Vietnam Journal of Mechanics, VAST, Vol.41, No. 2 (2019), pp. 193-201

DOI: https://doi.org/10.15625/0866-7136/13635

\title{
LOWER BOUND OF PERFORMANCE INDEX OF ANTI-SWAY CONTROL OF A PENDULUM USING ON-OFF DAMPING RADIAL SPRING-DAMPER
}

\author{
La Duc Viet ${ }^{1,3}$, Nguyen Trong Kien ${ }^{2,3, *}$ \\ ${ }^{1}$ Institute of Mechanics, VAST, Hanoi, Vietnam \\ ${ }^{2}$ Vinh University, Nghe An, Vietnam \\ ${ }^{3}$ Graduate University of Science and Technology, VAST, Hanoi, Vietnam \\ *E-mail: 1dviet@imech.vast.vn
}

Received: 20 January 2019 / Published online: 10 June 2019

\begin{abstract}
An on-off damping radial spring-damper can be attached to a pendulum to suppress its nonlinear sway motion. This paper studies the class of on-off damping controller, which switches the damping level from high to low and back at fixed times every quarter of period. Among this class, this paper shows the solution of the lower bound controller producing the lowest amplitude-frequency curve. The lower bound curve shows some fundamental natures of the system and gives some useful directions for a good on-off damping controller.

Keywords: Coriolis damping; anti-sway control; analytical optimization; on-off damping; optimal bound.
\end{abstract}

\section{INTRODUCTION}

Residual sway vibration of a pendulum is often met in pendulum-type structures such as ropeway gondola, crane, floating structures (e.g., ships or tension leg platforms) or spacecraft. The anti-sway control problem of a single pendulum is often approached by regulating the movement of the fulcrum point, which can be referred, for example, in a numerous studies on anti-sway control of crane [1].

However, this paper does not consider the approach of moving the fulcrum point. In some recent studies, we have introduced the approach of radial spring-damper to reduce the sway motion [2,3]. It works in the principle of nonlinear Coriolis damping [4]. Without adaptability and flexibility, the passive damping, however, has some shortcomings. For example, the too small passive damping can not prevent the resonance motion but the too large passive damping reduces the damper motion and dissipation energy. The on-off damping is the simplest way to improve the adaptability of the damping. The device producing on-off damping is much cheaper and easier to control than the one producing continuous state damping [5]. The on-off damping, as a method of vibration

(C) 2019 Vietnam Academy of Science and Technology 
control, has been studied widely from the point of view of both control strategies and implemented devices, see for example [6-9] and references therein.

The on-off damping strategies have been developed extensively but all the studied controllers are inapplicable to the problem in this paper because the damper's radial force is not codirectional with the sway motion. The novelty in this paper is a clear derivation of the lower bound of performance index of an on-off damping radial springdamper incorporating to a simple pendulum. This lower bound gives a useful analytical benchmark to evaluate any other practical controller.

\section{PROBLEM STATEMENT}

In $[2,3]$, the passive radial spring-damper was proposed to reduce the sway motion of a pendulum. In this paper, the on-off damping is considered as shown in Fig. 1. The mechanism of the radial spring-damper is as follows. The sway motion induces the centrifugal force. Due to the spring-damper connection, the radial motion is excited and produces the Coriolis damping, which reduces the sway motion. In the passive damper, the too large damping prevents the radial motion and then the Coriolis damping. Conversely, the too small damping leads to the resonance motion. The on-off damping can overcome the shortcomings of too high or too low damping.

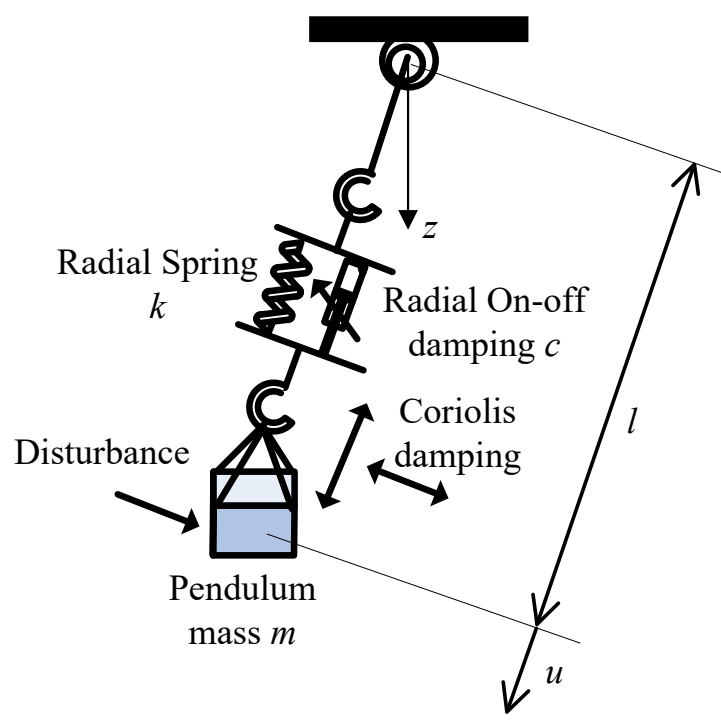

Fig. 1. Model of a single pendulum attached with radial spring and on-off damper

Let us denote $\theta$ as the sway angle, $u$ as the radial displacement (measured from static position). Denote $m$ as the pendulum mass, $k$ and $c$ respectively are the spring and damper coefficients, $l$ is the distances from the pendulum center of mass to the pivot in the static condition, $g$ is the acceleration of gravity. The structural damping is denoted by $c_{s}$. The external (non-gravitational) moment $M_{\theta}$ acts on the pendulum and is considered in the single harmonic form. The motion equations have been derived in [3] for 
the spherical pendulum. In this paper, we only consider the planar pendulum and the equations have the following non-dimensional form

$$
\begin{aligned}
& \left(1+u_{n}\right)^{2}\left(\ddot{\theta}+2 \zeta_{s} \dot{\theta}\right)+\left(1+u_{n}\right)\left(\sin \theta+2 \dot{u}_{n} \dot{\theta}\right)=2 \zeta_{s} \theta_{m} \cos \left(\beta \tau+\varphi_{f}\right), \\
& \ddot{u}_{n}+2 \zeta_{\dot{u}_{n}}+\alpha^{2} u_{n}-\left(1+u_{n}\right) \dot{\theta}^{2}+1-\cos \theta=0,
\end{aligned}
$$

in which

$$
\omega_{s}=\sqrt{\frac{g}{l}} ; \quad \zeta_{s}=\frac{c_{s}}{2 l^{2} m \omega_{s}} ; \quad \alpha=\frac{\sqrt{k / m}}{\omega_{s}} ; \quad \zeta=\frac{c}{2 m \omega_{s}} ; \quad u_{n}=\frac{u}{l} ; \quad \tau=\omega_{s} t,
$$

where $\omega_{s}$ is the natural frequency of pendulum, $\tau$ is the non-dimensional time with time scale $\omega_{s}^{-1}, \alpha$ is the ratio of two natural frequencies, $\zeta_{s}$ and $\zeta$ respectively are the damping ratios of the spherical pendulum and damper, $u_{n}$ is the non-dimensional form of radial movement. The dot operator from now denotes the differentiation with respect to normalized time $\tau$. The external moment $M_{\theta}$ have form

$$
M_{\theta}=2 \theta_{m} \zeta_{s} \omega_{s}^{2} m l^{2} \cos \left(\beta \tau+\varphi_{f}\right),
$$

where $\beta$ and $\varphi_{f}$ respectively are the excitation normalized frequency and phase, $\theta_{m}$ is the maximum sway angle of the linear pendulum (without spring and damper) at the resonance frequency.

Consider the on-off damping radial damper, i.e.

$$
\zeta=\left[\begin{array}{ll}
\zeta_{h} & \text { certain condition } \\
\zeta_{l} & \text { otherwise }
\end{array}\right.
$$

in which $\zeta_{h}$ and $\zeta_{l}$, respectively, are the on-value and off-value of the semi-active damping ratio. The damping is set to a value $\zeta_{h}$ when a certain condition is met and to a value $\zeta_{l}$ otherwise.

\section{LOWER BOUND SOLUTION}

In the single harmonic vibration, the switching times of many popular on-off controllers such as skyhook or ground hook controller are the roots of the product of two harmonic functions with the excitation frequency [10]. Therefore, normally, at a given frequency, the conventional controllers switch the damping level from high to low and back at fixed times every half period. The interesting lower bound solution for this class of on-off damping isolation system can be found in [11].

However, the conventional controllers are inapplicable to the problem in this paper because the damper force is not codirectional with the sway motion. In fact, as pointed out in $[2,3]$ the radial motion has the frequency of twice of the pendulum frequency. Because the damper force also follows this frequency, it is intuitive to consider the class of on-off damping, which switches the damping level from high to low and back at fixed times every quarter of period. The full nonlinear differential equation (1) is too complicated to derive the analytical solutions. Some simplifications can be used but retain the important natures of the system [3]. The order analysis is done by assuming the small 
values of sway angle, radial movement and damping ratio. Retaining the second order of $\theta, \dot{\theta}, \ddot{\theta}, u_{n}, \dot{u}_{n}, \ddot{u}_{n}, \zeta_{s}$ yields

$$
\begin{gathered}
\left(1+2 u_{n}\right) \ddot{\theta}+2\left(\zeta_{s}+\dot{u}_{n}\right) \dot{\theta}+\left(1+u_{n}\right) \theta=2 \zeta_{s} \theta_{m} \cos \left(\beta \tau+\varphi_{f}\right), \\
\ddot{u}_{n}+2 \zeta_{u_{n}}+\alpha^{2} u_{n}-\dot{\theta}^{2}+\frac{\theta^{2}}{2}=0 .
\end{gathered}
$$

The equation's forms (5), (6) are enough to derive the analytical solution but also retain the second order interaction between the sway motion and the radial motion.

The approximate solutions of the system response are sought in the form of harmonic functions as

$$
\theta=\theta_{0} \sin (\beta \tau), u_{n}=h \theta_{0}^{2}(\cos \phi \sin (2 \beta \tau)+\sin \phi \cos (2 \beta \tau))
$$

The three unknown $\theta_{0}, h$ and $\varphi$ satisfy the following conditions

$$
\theta_{0}>0 ; \quad h>0 ; \quad-\pi<\phi<\pi \text {. }
$$

Let us assume

$$
\zeta=\left[\begin{array}{ll}
\zeta_{l}, & \tau_{1}<\tau<\tau_{2} \\
\zeta_{h}, & \tau_{2}<\tau<\tau_{1}+\frac{\pi}{2 \beta}
\end{array}\right.
$$

In which, $\pi / 2 / \beta$ is the quarter of the (normalized) period of sway motion, $\tau_{1}$ and $\tau_{2}$ are two fixed switching (normalized) time at the given (normalized) frequency $\beta$. To present the results in a clearer form, let us denote

$$
\tau_{2}-\tau_{1}=\frac{\tau_{m}}{2 \beta} ; \quad \tau_{1}+\tau_{2}=\frac{\tau_{p}}{2 \beta} .
$$

The conditions of $\tau_{1}$ and $\tau_{2}$ shown in (9) also give the condition

$$
0 \leq \tau_{m} \leq \pi
$$

Use (9) and (10), some integrals relating to the on-off damping ratio are determined as

$$
\begin{aligned}
\int_{\tau_{1}}^{\tau_{1}+\frac{2 \pi}{\beta}} \zeta \mathrm{d} \tau= & 4 \int_{\tau_{1}}^{\tau_{1}+\frac{\pi}{2 \beta}} \zeta \mathrm{d} \tau=4\left(\zeta_{l}\left(\tau_{2}-\tau_{1}\right)+\zeta_{h}\left(\tau_{1}+\frac{\pi}{2 \beta}-\tau_{2}\right)\right)=\frac{2}{\beta}\left(\pi \zeta_{h}-\left(\zeta_{h}-\zeta_{l}\right) \tau_{m}\right) \\
& \int_{\tau_{1}}^{\tau_{1}+\frac{2 \pi}{\beta}} \zeta \cos (4 \beta \tau) \mathrm{d} \tau=4 \int_{\tau_{1}}^{\tau_{1}+\frac{\pi}{2 \beta}} \zeta \cos (4 \beta \tau) \mathrm{d} \tau \\
= & 4 \zeta_{l} \frac{\sin \left(4 \beta \tau_{2}\right)-\sin \left(4 \beta \tau_{1}\right)}{4 \beta}+4 \zeta_{h} \frac{\sin \left(4 \beta\left(\tau_{1}+\frac{\pi}{2 \beta}\right)\right)-\sin \left(4 \beta \tau_{2}\right)}{4 \beta} \\
= & -2 \frac{\zeta_{h}-\zeta_{l}}{\beta} \sin \tau_{m} \cos \tau_{p}
\end{aligned}
$$




$$
\int_{\tau_{1}}^{\tau_{1}+\frac{2 \pi}{\beta}} \zeta \sin (4 \beta \tau) \mathrm{d} \tau=-2 \frac{\zeta_{h}-\zeta_{l}}{\beta} \sin \tau_{m} \sin \tau_{p} .
$$

Substituting expression (7) into Eqs. (5)-(6) results in

$$
\begin{aligned}
& 2 \beta \zeta_{s} \theta_{0} \cos (\beta \tau)+\left(1-\beta^{2}\right) \theta_{0} \sin (\beta \tau) \\
& +\left(1-2 \beta^{2}\right) h \theta_{0}^{3} \sin (\beta \tau)(\cos \varphi \sin (2 \beta \tau)+\sin \varphi \cos (2 \beta \tau)) \\
& +4 h \beta^{2} \theta_{0}^{3} \cos (\beta \tau)(\cos \varphi \cos (2 \beta \tau)-\sin \varphi \sin (2 \beta \tau))=2 \zeta_{s} \theta_{m} \cos \left(\beta \tau+\varphi_{f}\right), \\
& 4 h \beta \theta_{0}^{2} \zeta(\cos \varphi \cos (2 \beta \tau)-\sin \varphi \sin (2 \beta \tau)) \\
& +h \theta_{0}^{2}\left(\alpha^{2}-4 \beta^{2}\right)(\cos \varphi \sin (2 \beta \tau)+\sin \varphi \cos (2 \beta \tau)) \\
& -\beta^{2} \theta_{0}^{2} \cos ^{2}(\beta \tau)+\theta_{0}^{2} \sin ^{2}(\beta \tau) / 2=0 .
\end{aligned}
$$

Multiply Eq. (15) with $\cos (\beta \tau)$ and $\sin (\beta \tau)$, respectively, then integrate over one period (from 0 to $2 \pi / \beta$ ), we have

$$
\begin{aligned}
& 2 \beta \zeta_{s} \theta_{0}+\left(\beta^{2}+\frac{1}{2}\right) h \theta_{0}^{3} \cos \varphi=2 \zeta_{s} \theta_{m} \cos \left(\varphi_{f}\right), \\
& \left(1-\beta^{2}\right) \theta_{0}-\left(\beta^{2}+\frac{1}{2}\right) h \theta_{0}^{3} \sin \varphi=-2 \zeta_{s} \theta_{m} \sin \left(\varphi_{f}\right) .
\end{aligned}
$$

Eliminating the excitation phase $\varphi_{f}$ between two equations of (17) gives a cubic equation of $\theta_{0}^{2}$ as

$$
\begin{aligned}
\left(\beta^{2}+\frac{1}{2}\right)^{2} h^{2} \theta_{0}^{6} & +2 h\left(\beta^{2}+\frac{1}{2}\right)\left(2 \zeta_{s} \beta \cos \varphi-\left(1-\beta^{2}\right) \sin \varphi\right) \theta_{0}^{4} \\
& +\left(4 \beta^{2} \zeta_{s}^{2}+\left(1-\beta^{2}\right)^{2}\right) \theta_{0}^{2}=4 \zeta_{s}^{2} \theta_{m}^{2}
\end{aligned}
$$

Multiply Eq. (16) with $\cos (2 \beta \tau)$ and $\sin (2 \beta \tau)$, respectively, then integrate over one period (from 0 to $2 \pi / \beta$ ), we have

$$
\begin{aligned}
& 2 h \beta \int_{\tau_{1}}^{\tau_{1}+\frac{2 \pi}{\beta}} \zeta(\cos \varphi(1+\cos (4 \beta \tau))-\sin \varphi \sin (4 \beta \tau)) \mathrm{d} \tau \\
& \quad+\left(\alpha^{2}-4 \beta^{2}\right) h \sin \varphi \frac{\pi}{\beta}-\left(\frac{2 \beta^{2}+1}{4}\right) \frac{\pi}{\beta}=0, \\
& \quad 2 \beta \int_{\tau_{1}}^{\tau_{1}+\frac{2 \pi}{\beta}} \zeta(\cos \varphi \sin (4 \beta \tau)-\sin \varphi(1-\cos (4 \beta \tau))) \mathrm{d} \tau+\left(\alpha^{2}-4 \beta^{2}\right) \cos \varphi \frac{\pi}{\beta}=0 .
\end{aligned}
$$


The integrals (12), (13) and (14) now are used in Eqs. (19) to gives

$$
\begin{aligned}
& 4 h\left(\pi \zeta_{h}-\left(\zeta_{h}-\zeta_{l}\right) \tau_{m}-\left(\zeta_{h}-\zeta_{l}\right) \cos \tau_{p} \sin \tau_{m}\right) \cos \phi \\
& +\left(4\left(\zeta_{h}-\zeta_{l}\right) \sin \tau_{p} \sin \tau_{m}+\left(\alpha^{2}-4 \beta^{2}\right) \frac{\pi}{\beta}\right) h \sin \phi-\left(\frac{2 \beta^{2}+1}{4}\right) \frac{\pi}{\beta}=0, \\
& -4\left(\pi \zeta_{h}-\left(\zeta_{h}-\zeta_{l}\right) \tau_{m}+\left(\zeta_{h}-\zeta_{l}\right) \cos \tau_{p} \sin \tau_{m}\right) \sin \phi \\
& -\left(4\left(\zeta_{h}-\zeta_{l}\right) \sin \tau_{p} \sin \tau_{m}-\left(\alpha^{2}-4 \beta^{2}\right) \frac{\pi}{\beta}\right) \cos \phi=0 .
\end{aligned}
$$

Totally we have 3 equations (18), (20) and (21) for 3 unknowns $\varphi, h$ and $\theta_{0}$. Moreover, $h$ and $\theta_{0}$ are absent in Eq. (21) while $\theta_{0}$ is absent in Eq. (20), that the equations can be solved sequentially.

The following steps are taken:

- Step 1: Solve (21) to obtain $\varphi$;

- Step 2: Substitute $\varphi$ to (20), then solve the obtained equation to find $h$;

- Step 3: Substitute $\varphi$ (found in step 1) and $h$ (found in step 2) to (18) to have the cubic equation of $\theta_{0}^{2}$ depending on two parameters $\tau_{p}$ and $\tau_{m}$;

- Step 4: Apply the minimization condition to solve $\tau_{P}$ as the function of $\theta_{0}$ and $\tau_{m}$.

After some manipulations shown in the Appendix, the final relation between $\theta_{0}$ and $\tau_{m}$ has following form

$$
a_{3}\left(\tau_{m}, \theta_{0}\right) \theta_{0}^{6}+a_{2}\left(\tau_{m}, \theta_{0}\right) \theta_{0}^{4}+a_{1} \theta_{0}^{2}+a_{0}=0,
$$

where

$$
\begin{gathered}
a_{3}=\frac{1}{4}\left(\beta^{2}+\frac{1}{2}\right)^{4} \frac{\pi^{2}}{\beta^{2}} \times \frac{\left(\alpha^{2}-4 \beta^{2}\right)^{2} \frac{\pi^{2}}{\beta^{2}}+16\left(\zeta_{1}^{2}+\zeta_{2}^{2}\right)+8 \zeta_{2}\left(4 \zeta_{1} \cos \tau_{p}-\sin \tau_{p}\left(\alpha^{2}-4 \beta^{2}\right) \frac{\pi}{\beta}\right)}{\left(\left(\alpha^{2}-4 \beta^{2}\right)^{2} \frac{\pi^{2}}{\beta^{2}}+16\left(\zeta_{1}^{2}-\zeta_{2}^{2}\right)\right)^{2}}, \\
a_{2}=\frac{\left(2 \beta^{2}+1\right)^{2} \pi}{4 \beta} \times \frac{8 \beta \zeta_{s} \zeta_{1}-\left(1-\beta^{2}\right)\left(\alpha^{2}-4 \beta^{2}\right) \frac{\pi}{\beta}+4 \zeta_{2}\left(\left(1-\beta^{2}\right) \sin \tau_{p}+2 \zeta_{s} \beta \cos \tau_{p}\right)}{\left(\alpha^{2}-4 \beta^{2}\right)^{2} \frac{\pi^{2}}{\beta^{2}}+16\left(\zeta_{1}^{2}-\zeta_{2}^{2}\right)}, \\
a_{1}=4 \beta^{2} \zeta_{s}^{2}+\left(1-\beta^{2}\right)^{2}, \\
\zeta_{0}=-4 \zeta_{s}^{2} \theta_{m}^{2}, \\
\tan \tau_{p}=\frac{\left(1-\tau_{m}\right)=\pi \zeta_{h}-\left(\zeta_{h}-\zeta_{l}\right) \tau_{m} ; \quad \zeta_{2}\left(\tau_{m}\right)=\left(\zeta_{h}-\zeta_{l}\right) \sin \tau_{m},}{\left.2 \beta\left(\beta^{2}\right)^{2} \frac{\pi^{2}}{\beta^{2}}+16\left(\zeta_{1}^{2}-\zeta_{2}^{2}\right)\right)-\frac{\left(\alpha^{2}-4 \beta^{2}\right) \pi^{2}\left(2 \beta^{2}+1\right)^{2} \theta_{0}^{2}}{8 \beta^{2}}}
\end{gathered}
$$

In brief, for a given (normalized) frequency $\beta$, the relation (22) gives a curve in the plane of $\theta_{0}$ and $\tau_{m}$. The lowest $\theta_{0}$ of the curve is the lower bound solution for the given frequency. Figs. 2 and 3 show some typical curves in the plane of $\theta_{0}$ and $\tau_{m}$ for some values of $\beta$. Other parameters are given as $\alpha=2, \zeta_{s}=1 \%, \theta_{m}=\pi / 6$. The parameter $\alpha$ is taken of 2 , which is near the optimal value of frequency ratio [2,3]. The on-off dampings $\zeta_{h}, \zeta_{l}$ are changed to see their effects. It is noted that two end values of $\tau_{m}$ correspond to 
the passive damping cases. When $\tau_{m}=0$, from (9) we have the passive high damping. Conversely, $\tau_{m}=\pi$ implies the passive low damping case.

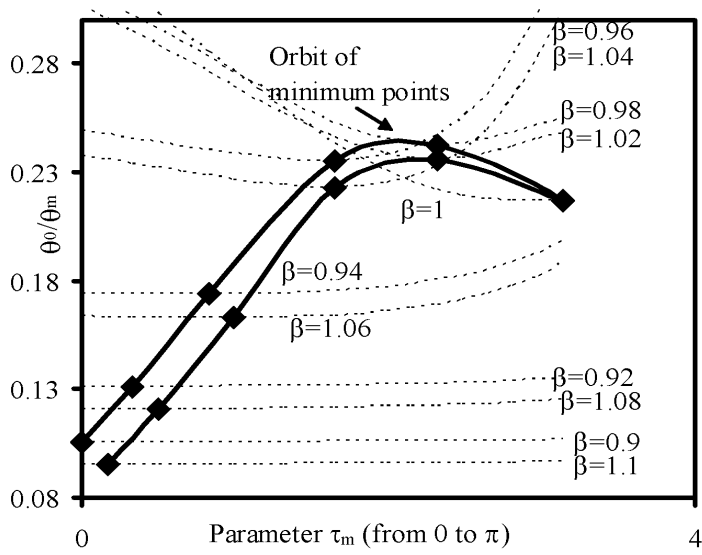

Fig. 2. Typical relation curves (dashed) between $\theta_{0}$ and $\tau_{m}$ and orbit (solid) of minimum points for $\zeta_{h}=20 \%, \zeta_{l}=5 \%, \alpha=2, \theta_{m}=\pi / 6$, $\zeta_{s}=1 \%$

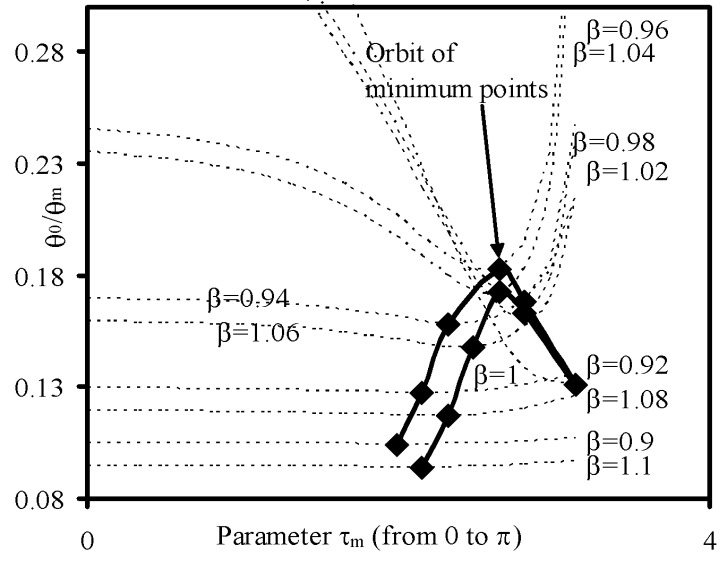

Fig. 3. Typical relation curves (dashed) between $\theta_{0}$ and $\tau_{m}$ and orbit (solid) of minimum points for $\zeta_{h}=100 \%, \zeta_{l}=1 \%, \alpha=2, \theta_{m}=\pi / 6$, $\zeta_{s}=1 \%$

The following observations of $\theta_{0}$ and $\tau_{m}$ can be drawn from the Figs. 2 and 3:

- The lower bound of sway angle amplitude $\theta_{0}$ increases when the excitation frequency ratio $\beta$ is near 1 . The behavior is clear because the resonance frequency ratio is 1 . Compare the orbits of minimum points between two Figs. 2 and 3, we see that the larger $\zeta_{h}$ and the smaller $\zeta_{l}$ give a lower value of lower bound.

- The optimal value of $\tau_{m}$ increases when the excitation frequency ratio $\beta$ is near 1 . Observe the on-off damping law (9), we see that increasing $\tau_{m}$ means the low damping is used longer. The optimal behavior means that when the frequency ratio $\beta$ is near 1 , the damping should be tuned longer to lower value to increase the radial motion to dissipate more energy. The outside region of $\beta$ requires the larger damping used more frequently to avoid the resonance motion (see the curves corresponding to $\beta=0.96$ and $\beta=1.04$ in both Figs. 2 and 3).

\section{CONCLUSIONS}

This paper considers the anti sway control of a single pendulum by an on-off damping radial spring-damper. For the single harmonic planar vibration, the theoretical solution of the lower bound of sway amplitude is derived. The lower bound is smaller with the larger on-damping and the smaller off-damping. The lower bound shows that when the forced frequency ratio is near 1, the damping should be tuned longer to lower value to increase the radial motion to dissipate more energy. The outside region of forced frequency ratio requires the larger damping used more frequently. 


\section{ACKNOWLEDGMENT}

This research is funded by Vietnam National Foundation for Science and Technology Development (NAFOSTED) under grant number 107.01-2015.35.

\section{REFERENCES}

[1] W. Singhose. Command shaping for flexible systems: A review of the first 50 years. International Journal of Precision Engineering and Manufacturing, 10, (4), (2009), pp. 153-168. https://doi.org/10.1007/s12541-009-0084-2.

[2] L. D. Viet. Crane sway reduction using Coriolis force produced by radial spring and damper. Journal of Mechanical Science and Technology, 29, (2015), pp. 973-979. https://doi.org/10.1007/s12206-015-0211-1.

[3] L. D. Viet. Partial stochastic linearization of a spherical pendulum with Coriolis damping produced by radial spring and damper. Journal of Vibration and Acoustics, 137, (5), (2015), p. 054504. https://doi.org/10.1115/1.4030663.

[4] L. D. Viet and N. B. Nghi. Approximated analytical frequency response of a pendulum structure attached with two orthogonal dynamic vibration absorbers. Vietnam Journal of Mechanics, 37, (4), (2015), pp. 275-284. https://doi.org/10.15625/0866-7136/37/4/5920.

[5] S. K. Yalla, A. Kareem, and J. C. Kantor. Semi-active tuned liquid column dampers for vibration control of structures. Engineering Structures, 23, (11), (2001), pp. 1469-1479. https://doi.org/10.1016/s0141-0296(01)00047-5.

[6] F. Casciati, G. Magonette, and F. Marazzi. Technology of semiactive devices and applications in vibration mitigation. John Wiley \& Sons, (2006).

[7] S. M. Savaresi, C. Poussot-Vassal, C. Spelta, O. Sename, and L. Dugard. Semi-active suspension control design for vehicles. Butterworth-Heinemann, UK, (2010).

[8] H. E. Tseng and D. Hrovat. State of the art survey: active and semi-active suspension control. Vehicle System Dynamics, 53, (7), (2015), pp. 1034-1062. https://doi.org/10.1080/00423114.2015.1037313.

[9] F. Casciati, J. Rodellar, and U. Yildirim. Active and semi-active control of structures: A review of recent advances. In Proceedings of the 8th International Conference on Structural Dynamics, EURODYN, Leuven, Belgium, (2011). pp. 62-69.

[10] V. D. La and C. Adam. General on-off damping controller for semi-active tuned liquid column damper. Journal of Vibration and Control, 24, (23), (2018), pp. 5487-5501. https://doi.org/10.1177/1077546316648080.

[11] J. N. Potter, S. A. Neild, and D. J. Wagg. Generalisation and optimisation of semi-active, onoff switching controllers for single degree-of-freedom systems. Journal of Sound and Vibration, 329, (13), (2010), pp. 2450-2462. https://doi.org/10.1016/j.jsv.2009.12.011. 


\section{APPENDIX}

This appendix derives the relation (22) between $\theta_{0}$ and $\tau_{m}$. Solving (21) gives

$$
\begin{aligned}
\sin \phi & = \pm \frac{\left(\alpha^{2}-4 \beta^{2}\right) \frac{\pi}{\beta}-4 \zeta_{2} \sin \tau_{p}}{\sqrt{\left(\alpha^{2}-4 \beta^{2}\right)^{2} \frac{\pi^{2}}{\beta^{2}}+16\left(\zeta_{1}^{2}+\zeta_{2}^{2}\right)+8 \zeta_{2}\left(4 \zeta_{1} \cos \tau_{p}-\sin \tau_{p}\left(\alpha^{2}-4 \beta^{2}\right) \frac{\pi}{\beta}\right)}} \\
\cos \phi & = \pm \frac{4\left(\zeta_{1}+\zeta_{2} \cos \tau_{p}\right)}{\sqrt{\left(\alpha^{2}-4 \beta^{2}\right)^{2} \frac{\pi^{2}}{\beta^{2}}+16\left(\zeta_{1}^{2}+\zeta_{2}^{2}\right)+8 \zeta_{2}\left(4 \zeta_{1} \cos \tau_{p}-\sin \tau_{p}\left(\alpha^{2}-4 \beta^{2}\right) \frac{\pi}{\beta}\right)}}
\end{aligned}
$$

where $\zeta_{1}$ and $\zeta_{2}$ are defined in (27). The $\operatorname{sign}$ of $\sin \varphi$ and $\cos \varphi$ must be chosen to satisfy the condition $h>0$ in (8). Substituting (29) into (20) gives

$$
\pm h \frac{16\left(\zeta_{1}^{2}-\zeta_{2}^{2}\right)+\left(\alpha^{2}-4 \beta^{2}\right)^{2} \frac{\pi^{2}}{\beta^{2}}}{\sqrt{\left(\alpha^{2}-4 \beta^{2}\right)^{2} \frac{\pi^{2}}{\beta^{2}}+16\left(\zeta_{1}^{2}+\zeta_{2}^{2}\right)+8 \zeta_{2}\left(4 \zeta_{1} \cos \tau_{p}-\sin \tau_{p}\left(\alpha^{2}-4 \beta^{2}\right) \frac{\pi}{\beta}\right)}}=\left(\frac{2 \beta^{2}+1}{4}\right) \frac{\pi}{\beta} .
$$

It is not difficult to check that $\zeta_{1}>\zeta_{2}$ when $\tau_{m}$ satisfies the condition (11). Therefore, to guarantee the condition $h>0$, the plus sign is used in (29) and (30). Substituting $\varphi$ from (29) and $h$ from (30) to (18), we obtain the equation (22) where the coefficients $a_{i}(i=0, \ldots, 3)$ are defined in (23)-(26). To eliminate the parameter $\tau_{P}$, we use the minimization condition. Taking the derivative of Eq. (22) with respect to $\tau_{P}$ gives

$$
\frac{\partial a_{3}}{\partial \tau_{P}} \theta_{0}^{6}+6 a_{3} \theta_{0}^{5} \frac{\partial \theta_{0}}{\partial \tau_{P}}+\frac{\partial a_{2}}{\partial \tau_{P}} \theta_{0}^{4}+4 a_{2} \theta_{0}^{3} \frac{\partial \theta_{0}}{\partial \tau_{P}}+2 a_{1} \theta_{0} \frac{\partial \theta_{0}}{\partial \tau_{P}}=0 .
$$

The minimization condition $\frac{\partial \theta_{0}}{\partial \tau_{P}}=0$ applying to (31) gives

$$
\frac{\partial a_{3}}{\partial \tau_{P}} \theta_{0}^{2}+\frac{\partial a_{2}}{\partial \tau_{P}}=0
$$

Using the forms of $a_{2}$ and $a_{3}$ in (23) reduces (24) to a trigonometric equation, which can be solved for $\tau_{P}$. The root obtained is shown in (28). 